\title{
Diversidad y distribución espacio-temporal de macroinvertebrados acuáticos en una charca temporaria urbana (Córdoba, Argentina)
}

\section{Diversity and spatio-temporal distribution of aquatic macroinvertebrates in an urban temporary pond (Córdoba, Argentina)}

\author{
María Soledad Abraham¹, Evangelina Sandra Natale ${ }^{1,2}$, Romina Elizabeth Principe ${ }^{1,2}$ \\ 1 Departamento de Ciencias Naturales, Universidad Nacional de Río Cuarto (UNRC). Ruta 36 Km 601 \\ X5804BYA, AP N³3, Río Cuarto, Córdoba, Argentina. \\ IInstituto de Ciencias de la Tierra, Biodiversidad y Ambiente (ICBIA) UNRC-CONICET \\ Consejo Nacional de Investigaciones Científicas y Técnicas.
}

E-mail: rprincipe@exa.unrc.edu.ar

\begin{abstract}
RESUMEN. Las charcas temporarias constituyen "puntos calientes" de biodiversidad dentro de una región o paisaje. Dada su importancia en el contexto paisajístico, el objetivo de este estudio fue evaluar la diversidad y composición del ensamble de macroinvertebrados acuáticos de una charca temporaria urbana, analizando su distribución espacial y temporal. Se recolectaron macroinvertebrados acuáticos en diferentes hábitats dentro de la charca durante período seco y húmedo, se identificaron y cuantificaron en el laboratorio y se asignaron a grupos en función de sus estrategias de vida. Se registraron 53 taxa de macroinvertebrados y el análisis de correspondencias diferenció el ensamble en función de la presencia de vegetación acuática y de la estacionalidad. Mediante el método del Valor Indicador se identificaron taxa característicos de los distintos hábitats y períodos hidrológicos. El Grupo 1 de estrategia de vida (sobreviven a la desecación, requieren agua para la reproducción y tienen dispersión pasiva) y el Grupo 4 (no sobreviven a la desecación, requieren agua para la reproducción y tienen dispersión activa) presentaron mayor número de taxa en todos los hábitats. La mayor abundancia se registró en los sedimentos sin vegetación mientras que la riqueza, la diversidad y la equitatividad fueron mayores en hábitats vegetados. Los resultados destacan la importancia de las macrófitas y la estacionalidad en el mantenimiento de la biodiversidad y proporcionan importante información de base para el monitoreo, manejo y conservación de charcas temporarias urbanas.
\end{abstract}

Palabras clave: Estacionalidad, estrategias de historia de vida, hábitat, invertebrados acuáticos, macrófitas.

ABSTRACT. Temporary ponds are worldwide recognized as 'hotspots' of biodiversity within a region or landscape. The aim of this study was to assess the diversity and composition of the aquatic macroinvertebrate assemblages of an urban temporary pond, due to its importance in the landscape context. We analized the spatial distribution of the assemblages in different habitats and their temporal variation. Aquatic macroinvertebrates were collected in wet and dry periods by sampling scoops and hand net. The specimens were identified, quantified, and assigned to groups considering their life-history strategies. Fifty-three invertebrate taxa were identified and the correspondence analysis distinguished the assemblages in relation to the presence of aquatic vegetation and seasonality. The Indicator Value method identified characteristic taxa of the different habitats and hydrological periods. The Group 1 of life-history strategy (with adaptations to desiccation, water requirement for reproduction and with passive dispersion) and the Group 4 (without adaptations to desiccation, water requirement for reproduction and with active dispersion) presented the highest number of taxa in all habitats. The highest abundance was observed in 
sediments without vegetation whereas richness, diversity and evenness were higher in vegetated habitats. These results highlight the importance of macrophytes and seasonality in the maintenance of biodiversity and provide baseline information for monitoring, management and conservation of urban temporary ponds.

Keywords: Aquatic invertebrates, habitat, life-history strategies, macrophytes, seasonality.

\section{INTRODUCCIÓN}

Las charcas temporarias desempeñan un papel esencial como proveedoras de servicios ambientales, ofreciendo soluciones sostenibles para algunos de los problemas clave del cambio climático y la gestión del agua (European Pond Conservation Network, 2008). A nivel de paisaje, son especialmente importantes ya que constituyen corredores biológicos que incrementan la conectividad entre hábitats de agua dulce y contribuyen a la biodiversidad regional, tanto como los ríos o los lagos (Williams et al., 2004; Céréghino et al., 2008).

Dado que las charcas en las zonas urbanas se utilizan con frecuencia para controlar el flujo de aguas pluviales (Mitsch \& Gosselink, 2000), en general no suelen incluirse en trabajos de investigación ni se las considera como áreas importantes para la conservación (Hassall, 2014). Sin embargo, estos humedales urbanos pueden sustentar una elevada biodiversidad, proveer servicios ambientales y contribuir a la estética del paisaje (Noble \& Hassall, 2015). La presencia de estos espacios verdes en las ciudades no sólo desempeña un papel considerable en la mejora del bienestar individual y comunitario (Lee \& Maheswaran, 2011) sino que, además, es una importante oportunidad para educar a la sociedad sobre los sistemas naturales y la biodiversidad en general (Williams et al., 2004; Hassall, 2014).

Según la Convención de Ramsar (2002), se define como charca a una masa de agua estancada de hasta 10 hectáreas, con profundidad, estacionalidad y origen diverso (natural o artificial). Básicamente se caracterizan por alternar fases de inundación y de sequía que suelen ser impredecibles en su forma y duración (Williams, 1997). En las charcas temporarias la permanencia del agua es una de las variables que más influye sobre las comunidades acuáticas
(Johansson \& Suhling, 2004), siendo también importantes la estructura del hábitat, el área de la charca, la riqueza de especies de macrófitas y las variables fisicoquímicas (Bazzanti et al., 1996; Boix et al., 2016; Jeffries, 2003; Williams, 1997). Todos estos factores ambientales delimitan características particulares en los cuerpos de agua temporarios determinando, en consecuencia, el establecimiento de comunidades de plantas y animales exclusivas y diversas (European Pond Conservation Network, 2008; Bazzanti et al., 2010).

Dentro de los grupos faunísticos que habitan cuerpos de agua temporarios, los invertebrados cumplen un papel esencial en la estructura y función de estos ecosistemas (Williams, 1997) y son utilizados de manera creciente como bioindicadores de la calidad del agua (Barbour et al., 1995; Boix et al., 2005; Nieto et al., 2017). Muchas especies de invertebrados que viven en las charcas temporarias tienen morfologías, historias de vida o mecanismos de dispersión que les permiten sobrevivir al ciclo de desecación que éstas presentan (Williams, 1997).

En el campus de la Universidad Nacional de Río Cuarto (provincia de Córdoba, Argentina) se localiza una charca temporaria de origen artificial lindante a un área protegida, la Reserva Natural Urbana Bosque Autóctono "El Espinal". Ambos se encuentran inmersos en una matriz urbana por lo que adquieren importancia como corredores biológicos al conectar parches aislados con los ecosistemas ribereños del río Chocancharava, curso de agua que atraviesa la trama urbana. Dada la importancia del cuerpo de agua en el contexto paisajístico, el objetivo de estudio fue evaluar la diversidad y composición del ensamble de macroinvertebrados acuáticos asociados a la charca, analizando su distribución espacial en diferentes hábitats y su variación temporal 
entre período seco y húmedo. Dado que la fauna de invertebrados acuáticos depende de las precipitaciones y de las características de los ambientes dentro de la charca, se espera que la diversidad y composición del ensamble varíe entre los diferentes hábitats y entre los períodos de lluvia y sequía.

\section{MATERIALES Y MÉTODOS Área de estudio}

El área de estudio comprendió una charca de carácter temporario ubicada en el campus de la Universidad Nacional de Río Cuarto, provincia de Córdoba, Argentina ( $33^{\circ} 6^{\prime} \mathrm{S}$, $64^{\circ} 18^{\prime}$ O, 420 m s.n.m.). La zona inundable del cuerpo de agua corresponde a una porción de paleocauce del Río Chocancharava (subcuenca Carcarañá), actualmente a una distancia de 800 $\mathrm{m}$. Asimismo, a $100 \mathrm{~m}$ de la charca se localiza un área protegida, la cual constituye un pequeño relicto representativo del distrito biogeográfico del Espinal (Morrone, 2014), cuya vegetación dominante es el bosque xerófilo caducifolio. Actualmente la mayor parte de este territorio está cubierto por tierras cultivadas y por campos de pastoreo con escasos relictos de vegetación natural (Cabido et al., 2003). La superficie del campus de la Universidad posee a nivel de paisaje una configuración en mosaico con parches de vegetación natural y seminatural, inmersos en una matriz urbana-rural. Los márgenes de la charca están constituidos por parches de vegetación exótica ornamental y vegetación autóctona.

El relieve forma parte de la Planicie Pampeana que caracteriza las regiones centrales de Argentina y el clima es templado con inviernos secos. La temperatura media anual es de $16,5^{\circ} \mathrm{C}$ y las precipitaciones oscilan entre 500 y 600 $\mathrm{mm}$ anuales, concentrándose entre octubre y marzo (Cabido et al., 2003). El volumen de la charca depende del aporte de sistemas de escurrimiento pluvial los cuales evacuan excedentes de un área de aproximadamente 800 ha. Estos excedentes pluviales se almacenan parcialmente en la charca, conformando así el sistema estacional.

\section{Muestreos y análisis de laboratorio}

Se recolectaron muestras demacroinvertebrados acuáticos en cada uno de los diferentes ambientes identificados dentro de la charca durante el año 2010 en período seco (junio) y húmedo (diciembre). En el período de bajas precipitaciones se identificaron seis hábitats para el muestreo: 1) parche de la macrófita emergente Polygonum aviculare L. de $38,5 \mathrm{~m}^{2}$; 2) parche de la macrófita flotante Heteranthera multiflora (Griseb.) Horn de $10 \mathrm{~m}^{2}$; 3) parche de la macrófita flotante Pistia stratiotes L. de $17,5 \mathrm{~m}^{2}$; 4) acumulaciones de macroalgas (Oedogonium sp.) de $6 \mathrm{~m}^{2}$; 5) sedimento sin vegetación y 6) sector de descarga del desagüe pluvial que alimenta la charca. En el período húmedo se pudieron incluir solo tres de los ambientes analizados en el período seco: 1) parche de la macrófita emergente $P$. aviculare de $15 \mathrm{~m}^{2}, 2$ ) sedimento sin vegetación y 3 ) sector de descarga del desagüe; ya que $P$. stratiotes, $H$. multiflora y acumulaciones de macroalgas no se registraron en la charca. En cada hábitat se tomaron dos réplicas al azar.

Para la recolección de invertebrados en parches de macrófitas y macroalgas se utilizó un cucharón muestreador de $500 \mathrm{ml}$ (diámetro $0,13 \mathrm{~m}$ ) y una red de mano (copo entomológico) de $300 \mu \mathrm{m}$ de abertura de malla (diámetro $0,25 \mathrm{~m}$ ). Con el cucharón se recogieron $10 \mathrm{~L} \mathrm{de}$ agua entre los parches los cuales se filtraron a través de la red de mano para retener allí los invertebrados de cada muestra (10 L por muestra). A medida que se recolectaba el agua con el cucharón, los parches de macrófitas y macroalgas eran sacudidos para lograr el desprendimiento de los invertebrados. Este procedimiento también se utilizó para el muestreo del sector de descarga del desagüe, ya que éste estuvo completamente cubierto de macrófitas en ambos períodos hidrológicos.

En el hábitat de sedimento sin vegetación se recolectó sedimento del fondo de la charca con cucharón muestreador de $150 \mathrm{ml}$ (diámetro 0,08 m). Para cada muestra se recolectó un cucharón y la totalidad del sedimento recogido se colocó en un frasco para su procesamiento en el laboratorio. Las muestras fueron fijadas 
con solución de formaldehido $4 \%$ y preservadas en solución de etanol $70 \%$. El procesamiento de las muestras se llevó a cabo en el laboratorio y la identificación de los invertebrados se realizó hasta el menor nivel taxonómico posible, mediante el uso de claves especializadas (Lopretto y Tell, 1995; Domínguez y Fernández, 2009). Los organismos de todas las muestras se cuantificaron como número de individuos por unidad de volumen y se incorporaron a la colección de Invertebrados del Departamento de Ciencias Naturales de la UNRC (Córdoba, Argentina).

De acuerdo con la clasificación propuesta por Wiggins et al. (1980), los invertebrados se clasificaron en cuatro grupos, en función de sus estrategias de vida. El Grupo 1 (G1) estuvo conformado por especies que sobreviven a la desecación, tienen dispersión pasiva y necesitan agua para la reproducción; el Grupo 2 (G2) incluyó especies que sobreviven a la desecación, tienen dispersión activa y necesitan agua para la reproducción; el Grupo 3 (G3) estuvo conformado por organismos que sobreviven a la desecación, tienen dispersión activa y no necesitan agua para la reproducción y el Grupo 4 (G4) incluyó especies que no sobreviven a la desecación, tienen dispersión activa y necesitan agua para la reproducción.

Para caracterizar el cuerpo de agua, se midieron variables fisicoquímicas, como temperatura (termómetro digital HANNA H198501), pH y conductividad (mediante sensores portátiles HANNA H198103 y H19803, respectivamente). En laboratorio, se determinaron sólidos disueltos totales (SDT), carbonatos, bicarbonatos, sulfatos, cloruros, sodio, potasio, calcio, magnesio, nitratos, nitritos, fluoruros, dureza total y alcalinidad de las muestras de agua de acuerdo con métodos propuestos por APHA (1998).

Con el fin de analizar el contenido de materia orgánica y caracterizar el sustrato se tomaron muestras de sedimentos en cada uno de los ambientes considerados para la colecta de invertebrados durante el período seco. La materia orgánica presente en cada muestra se determinó por diferencia de peso utilizando una balanza analítica con precisión de 0,001 g, luego de haber sido tratada con peróxido de hidrógeno $\left(\mathrm{H}_{2} \mathrm{O}_{2}\right)$ al $30 \%$ para la oxidación de la materia orgánica. Para analizar la composición del sustrato se determinó el porcentaje en peso de cada clase textural de acuerdo con la escala de Wentworth (1922), aplicando el método de tamizado. Se apilaron en orden ascendente tamices con diferente abertura de malla (grava fina: $4 \mathrm{~mm}$, grava muy fina: 2 $\mathrm{mm}$, arena muy gruesa: $1 \mathrm{~mm}$, arena gruesa: $1 / 2 \mathrm{~mm}$, arena media: $1 / 4 \mathrm{~mm}$, arena fina: $1 / 8$ $\mathrm{mm}$, arena muy fina: $1 / 16 \mathrm{~mm}$ y limo grueso: $1 / 32 \mathrm{~mm}$ ). Por debajo se colocó una base que recolecta "pelitas" (1/64 $\mathrm{mm})$, que incluye limo y arcilla. Luego, mediante la utilización de un agitador mecánico tipo Ro-Tap, la muestra se fraccionó completamente y se determinó el porcentaje de cada fracción a partir del peso del material retenido en cada malla y en la base, empleando balanza analítica con precisión de 0,001 g. Finalmente, para evaluar los cambios estacionales en la superficie de la charca se realizó el track del perímetro con GPS (Garmin etrex). Las mediciones se realizaron mensualmente durante el año en que se recolectaron las muestras (2010) y se incorporaron luego a un SIG (Arcmap 10.1) para calcular el área.

\section{Análisis de datos}

Para evaluar la variación espacio-temporal de los ensambles de invertebrados se realizó un Análisis de Correspondencia (AC) utilizando CANOCO versión 4.02 (ter Braak \& Smilauer, 1998). Los taxones de macroinvertebrados característicos de cada ambiente de la charca y de cada período hidrológico se obtuvieron mediante el Método del Valor Indicador (IndVal Method, Dufrêne \& Legendre, 1997) utilizando el programa estadístico PC-Ord for Windows 4.25 (McCune \& Mefford, 1999). Los taxa con valores de IndVal superiores a $70 \%$ se consideraron indicadores.

Para comparar el número de taxones pertenecientes a cada estrategia de vida entre los distintos hábitats se realizaron ANOVAs de una vía (factor hábitat) considerando separadamente los datos correspondientes a cada período hidrológico. Adicionalmente, la comunidad de macroinvertebrados se caracterizó a través del cálculo de abundancia, riqueza taxonómica y de los índices de diversidad $\left(\mathrm{H}^{\prime}\right)$ y equitatividad $\left(J^{\prime}\right)$ de Shannon para cada muestra. Estos 
atributos estructurales se compararon mediante ANOVAs de dos vías para poder evaluar la interacción entre los factores: factor período hidrológico (seco y húmedo) y factor hábitat (Polygonum aviculare, fondo sin vegetación y descarga del desagüe). En todos los ANOVAs, el supuesto de normalidad fue verificado mediante el test Shapiro-Wilk e histogramas y el de homogeneidad de varianzas a través del análisis de diagramas de dispersión de residuos vs. predichos. Los datos de abundancia y riqueza taxonómica se transformaron a log 10 (Y) y $\ln (Y)$ respectivamente, para cumplir con los supuestos requeridos por el análisis. Para las comparaciones a posteriori se utilizó la prueba DGC (Di Rienzo - Guzman - Casanoves, $\mathrm{a}=0,05$ ), un método jerárquico que controla el error de tipo I manteniendo una potencia aceptable ( $\mathrm{Di}$ Rienzo et al., 2002). Los análisis de varianza se realizaron utilizando el programa INFOSTAT Versión 2010 (Di Renzo et al., 2010).

\section{RESULTADOS}

Los valores de las variables químicas fueron diferentes entre los períodos hidrológicos siendo, en general, más elevados en el período seco (Tabla 1). El agua se clasificó como moderadamente dura y bicarbonatada cálcica y los valores de nitratos y fluoruros no superaron los límites establecidos en la normativa de calidad de agua para consumo humano, según el Código Alimentario Argentino (Ley 18 284, Decreto 2126/71, Capítulo XII). En el período húmedo, la temperatura del agua fue levemente mayor y la profundidad promedio presentó valores más elevados y mayores diferencias entre hábitats. La superficie de la charca alcanzó su máximo valor en el mes de octubre $\left(9807,5 \mathrm{~m}^{2}\right)$ inmediatamente después del inicio de período lluvioso, luego de haber permanecido sin agua durante el mes de septiembre (Figura 1). El porcentaje de materia orgánica en el sedimento varió entre 1,9\% y $4,4 \%$, registrándose los menores porcentajes en el sedimento debajo de la macrófita flotante $P$. stratiotes y en el sedimento sin vegetación, y el mayor valor en el sedimento de la macrófita emergente $P$. aviculare. En general, los hábitats vegetados ubicados en los márgenes de la charca (acumulaciones de macroalgas, $H$. multiflora y $P$. aviculare), presentaron mayores porcentajes de materia orgánica que los ambientes situados hacia el centro del cuerpo de agua (sector desagüe, fondo sin vegetación y $P$. stratiotes).

Con respecto al análisis granulométrico del sedimento, se observó que las muestras colectadas en el sector del desagüe, en acumulación de macroalgas, en $P$. stratiotes y en el sedimento sin vegetación presentaron una mayor heterogeneidad de clastos, abarcando granulometrías del tipo sábulo hasta pelitas

Tabla 1. Valores de las variables físicas y químicas registradas en la charca temporaria durante período seco y húmedo. Se incluyen los valores medidos de profundidad de cada uno de los hábitats ( $\mathrm{Pa}$ : Polygonum aviculare, Hm: Heteranthera multiflora, Ps: Pistia stratiotes, M: macroalgas, S: sedimento sin vegetación, D: sector descarga del desagüe.

Table 1. Values of physical and chemical variables registered in the temporary pond during the dry and wet period. The measured depth values at each analyzed habitat are included (Pa: Polygonum aviculare, Hm: Heteranthera multiflora, Ps: Pistia stratiotes, M: macroalgae, S: sediment without vegetation, D: drain discharge sector).

\begin{tabular}{lcc} 
& $\begin{array}{c}\text { Período } \\
\text { seco }\end{array}$ & $\begin{array}{c}\text { Período } \\
\text { húmedo }\end{array}$ \\
\hline Temperatura del agua $\left({ }^{\circ} \mathrm{C}\right)$ & 14,3 & 16,0 \\
$\mathrm{pH}$ & 7,75 & 7,24 \\
Conductividad $25^{\circ} \mathrm{C}(\mu \mathrm{S} / \mathrm{cm})$ & 341 & 184 \\
Sólidos Disueltos Totales & 282 & 185 \\
(mg/l) & 170,0 & 112,5 \\
Bicarbonatos $(\mathrm{mg} / \mathrm{l})$ & 11,8 & 11,8 \\
Sulfatos $(\mathrm{mg} / \mathrm{l})$ & 11,4 & 11,4 \\
Cloruros $(\mathrm{mg} / \mathrm{l})$ & 22,2 & 4,0 \\
Sodio $(\mathrm{mg} / \mathrm{l})$ & 8,5 & 6,2 \\
Potasio $(\mathrm{mg} / \mathrm{l})$ & 39,2 & 28,0 \\
Calcio $(\mathrm{mg} / \mathrm{l})$ & 7,3 & 3,4 \\
Magnesio $(\mathrm{mg} / \mathrm{l})$ & 11,0 & 7,0 \\
Nitrato $(\mathrm{mg} / \mathrm{l})$ & 0,4 & 0,2 \\
Fluoruro $(\mathrm{mg} / \mathrm{l})$ & 2,6 & 2,3 \\
Dureza total $(\mathrm{meq} / \mathrm{l})$ & 2,7 & 1,8 \\
Alcalinidad & $0,10(\mathrm{~Pa})$ & 0,22 \\
\hline & $0,21(\mathrm{~Pa})$ \\
& $0,10(\mathrm{Ps})$ & $0,12(\mathrm{~S})$ \\
& $0,12(\mathrm{M})$ & \\
Profundidad promedio $(\mathrm{m})$ & $0,18(\mathrm{~S})$ & \\
& $0,25(\mathrm{D})$ & \\
\hline & & \\
& &
\end{tabular}




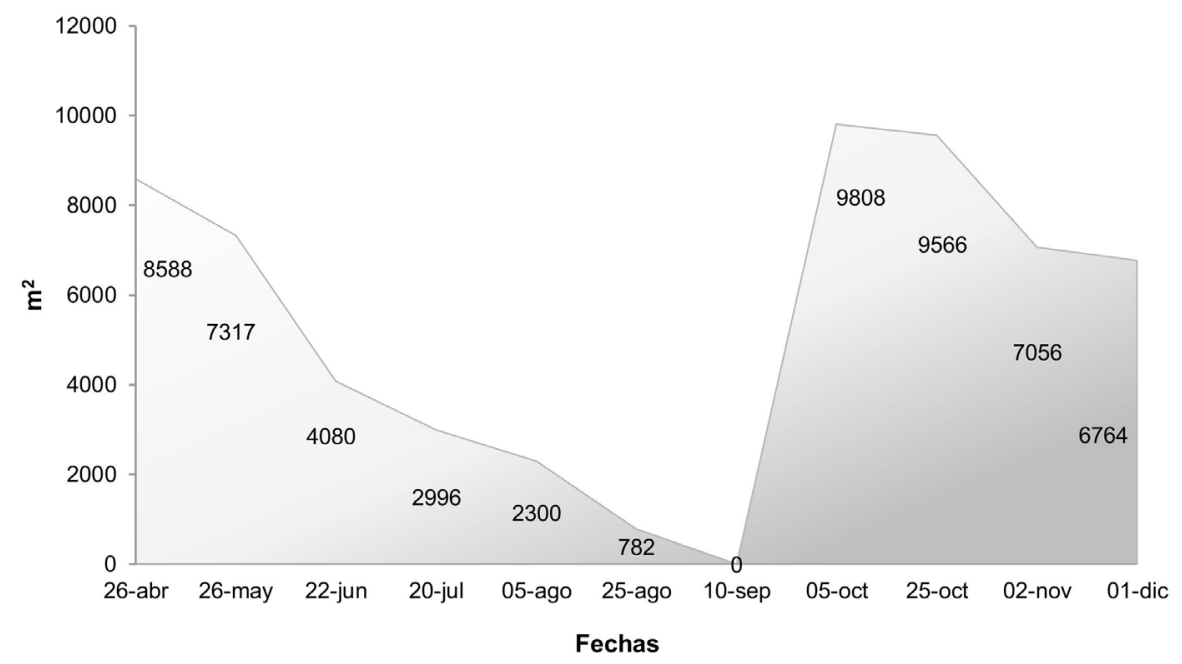

Figura 1. Variación mensual de la superficie de la charca temporaria en $\mathrm{m}^{2}$ durante el año 2010 (Río Cuarto, Córdoba). Los muestreos de invertebrados acuáticos se realizaron en los meses de junio y diciembre de ese año.

Figure 1. Monthly variation of the temporary pond surface in $\mathrm{m}^{2}$ during 2010 (Río Cuarto, Córdoba). Sampling of aquatic invertebrates was carried out in June and December of that year.

(limo medio, fino y arcillas). Los mayores porcentajes en estos ambientes se registraron en arenas finas (28\%) y muy finas (30\%). Las muestras de sedimento correspondientes a los hábitats $H$. multiflora y $P$. aviculare presentaron una menor heterogeneidad de clastos, siendo las arenas gruesas (24\%), medias (42\%) y finas (28\%) las clases texturales con mayor abundancia.

Se identificaron 53 taxa de macroinvertebrados correspondientes a ocho Phyla (Tabla 2). Nematoda y Naidinae (Annelida) fueron los taxa que presentaron una mayor abundancia y frecuencia de aparición (100\%); sin embargo Insecta presentó la mayor riqueza taxonómica (35 taxas). El Análisis de Correspondencias mostró una separación de las muestras en función del hábitat y del período hidrológico (Figura 2). El Eje 1 separó las muestras recolectadas en el fondo sin vegetación del resto de los hábitats, indicando una segregación de los hábitats en relación con la presencia de vegetación acuática; mientras que el Eje 2 separó las muestras del período húmedo de las del período seco. Los autovalores de los ejes 1 y 2 fueron 0,394 y 0,290 respectivamente y estos explicaron el $32,3 \%$ de la varianza acumulada de los datos.
En el período seco se observó una fuerte dominancia de anélidos en los hábitats $P$. aviculare, $P$. stratiotes y acumulación de macroalgas (Figura 3), seguido por los nematodos como taxón subdominante; mientras que en el fondo sin vegetación esta dominancia se invirtió. El hábitat bajo la descarga del desagüe presentó una gran abundancia de insectos y fuertemente asociado con $H$. multiflora se registró el tardígrado Dactylobiotus parthenogeneticus Bertolani, 1982. En el período húmedo se observó una fuerte dominancia de anélidos en todos los hábitats principalmente en el fondo sin vegetación (Figura 3). En P. aviculare se registraron también artrópodos y nematodos en el sector de descarga del desagüe.

El método IndVal permitió identificar taxa característicos de cada uno de los hábitats del período seco (Tabla 3). Los hábitats $P$. stratiotes y fondo sin vegetación no registraron taxa indicadores. En el período húmedo también se observaron diferencias entre hábitats (Tabla 3). Con respecto a los taxa característicos de cada período hidrológico, el método IndVal mostró que Nematoda presentó mayor valor indicador en el período seco registrándose cinco taxa indicadores en total para este período, con valores indicadores entre 99 y 
Tabla 2. Taxa de macroinvertebrados colectados en diferentes hábitats de una charca temporaria urbana (Córdoba, Argentina) durante el período seco (Se) y húmedo (Hu). Hábitats: Polygonum aviculare (Pa); Heteranthera multiflora $(\mathrm{Hm})$; Pistia stratiotes (Ps); macroalgas (M); sedimento sin vegetación (S) y sector de descarga del desagüe (D). Grupos de estrategia de vida (GEV) definidos en la sección de Materiales y Métodos. F: frecuencia de aparición (\%).

Table 2. Macroinvertebrates taxa collected in different habitats of an urban temporary pond (Córdoba, Argentina) during the dry (Se) and wet period (Hu). Habitats: Polygonum aviculare (Pa); Heteranthera multiflora $(\mathrm{Hm})$; Pistia stratiotes (Ps); macroalgae (M); sediment without vegetation (S) and drain discharge sector (D). Life strategy groups (EV) defined in the Materials and Methods section. F: occurrence frequency (\%).

\begin{tabular}{|c|c|c|c|c|c|}
\hline Phylum & TAXA & $F(\%)$ & Hábitats & Período & GEV \\
\hline Cnidaria & Hydra sp. & 22 & $\mathrm{Hm}-\mathrm{Ps}-\mathrm{M}$ & Se & G1 \\
\hline Platyhelminthes & Dugesiidae & 6 & $\mathrm{~Pa}$ & $\mathrm{Hu}$ & G1 \\
\hline Nemertea & Prostoma sp. & 17 & Hm-Ps-M & $\mathrm{Se}$ & G1 \\
\hline Nematoda & & 100 & Pa-S-D-Hm-Ps-M & $\mathrm{Se}-\mathrm{Hu}$ & G1 \\
\hline Tardigrada & Dactylobiotus partenogeneticus Bertolani, 1982 & 17 & $\mathrm{Hm}-\mathrm{M}$ & $\mathrm{Se}$ & G1 \\
\hline Mollusca & Lymnaea sp. & 17 & $\mathrm{Hm}-\mathrm{Ps}-\mathrm{M}$ & $\mathrm{Se}$ & G1 \\
\hline \multirow[t]{12}{*}{ Annelida } & Naidinae & 100 & Pa-S-D-Hm-Ps-M & $\mathrm{Se}-\mathrm{Hu}$ & G1 \\
\hline & Pristina sp. & 50 & Pa-D-Hm-Ps-M & $\mathrm{Se}$ & G1 \\
\hline & Chaetogaster sp. & 17 & Pa-M-D & Se-Hu & G1 \\
\hline & Dero (Aulophorus) sp. & 33 & Hm-P-Pa-S-D & $\mathrm{Se}-\mathrm{Hu}$ & G1 \\
\hline & Dero (Dero) sp. & 6 & $\mathrm{~S}$ & $\mathrm{Hu}$ & G1 \\
\hline & Stylaria sp. & 17 & $M-D$ & Se & G1 \\
\hline & Tubificinae & 17 & M-S-D & $\mathrm{Se}-\mathrm{Hu}$ & G1 \\
\hline & Lumbriculidae & 6 & $\mathrm{M}$ & Se & G1 \\
\hline & Lumbricidae & 11 & S-D & $\mathrm{Hu}$ & G1 \\
\hline & Hirudinea (morfotipo 1) & 67 & Pa-Hm-Ps-M-D-S & $\mathrm{Se}-\mathrm{Hu}$ & G1 \\
\hline & Hirudinea (morfotipo 2) & 39 & Pa-Hm-Ps-M & $\mathrm{Se}$ & G1 \\
\hline & Hirudinea (morfotipo 3) & 17 & $\mathrm{Hm}-\mathrm{Ps}-\mathrm{S}$ & $\mathrm{Se}$ & G1 \\
\hline \multirow[t]{34}{*}{ Arthropoda } & Hydrachnidia & 39 & Hm-M- D-Pa & $\mathrm{Se}-\mathrm{Hu}$ & G4 \\
\hline & Ostracoda & 50 & $\mathrm{Hm}-\mathrm{Ps}-\mathrm{M}-\mathrm{S}-\mathrm{Pa}-\mathrm{D}$ & $\mathrm{Se}-\mathrm{Hu}$ & G1 \\
\hline & Isotomidae & 67 & Hm-P- M-Pa-D & $\mathrm{Se}-\mathrm{Hu}$ & G1 \\
\hline & Poduridae & 28 & Hm-M-D & $\mathrm{Se}-\mathrm{Hu}$ & G1 \\
\hline & Entomobryidae & 33 & $\mathrm{Hm}-\mathrm{M}-\mathrm{D}-\mathrm{Pa}$ & $\mathrm{Se}-\mathrm{Hu}$ & G1 \\
\hline & Sminthuridae & 39 & $\mathrm{Hm}-\mathrm{M}-\mathrm{D}-\mathrm{Pa}$ & $\mathrm{Se}-\mathrm{Hu}$ & G1 \\
\hline & Callibaetis sp. & 6 & $\mathrm{~Pa}$ & Se & G4 \\
\hline & Camelobaetidius penai Traver \& Edmunds, 1968 & 6 & Ps & $\mathrm{Se}$ & G4 \\
\hline & Coeangrionidae & 72 & Hm-Ps-M-Pa-D & $\mathrm{Se}-\mathrm{Hu}$ & G4 \\
\hline & Lestidae & 17 & $\mathrm{Hm}-\mathrm{M}$ & $\mathrm{Se}$ & G3 \\
\hline & Gomphidae & 11 & $\mathrm{Hm}-\mathrm{Ps}$ & Se & G4 \\
\hline & Aeshnidae & 17 & $\mathrm{Hm}-\mathrm{Ps}$ & $\mathrm{Se}$ & G4 \\
\hline & Libellulidae & 6 & M & Se & G4 \\
\hline & Belostoma sp. & 39 & $\mathrm{Hm}-\mathrm{M}-\mathrm{Pa}-\mathrm{D}$ & $\mathrm{Se}-\mathrm{Hu}$ & G4 \\
\hline & Belostomatidae (ninfas) & 11 & $\mathrm{~Pa}$ & $\mathrm{Hu}$ & G4 \\
\hline & Buenoa sp. & 17 & $M-D$ & $\mathrm{Se}$ & G4 \\
\hline & Notonecta sp. & 11 & Ps-M & $\mathrm{Se}$ & G4 \\
\hline & Notonectidae (ninfas) & 6 & $\mathrm{Hm}$ & Se & G4 \\
\hline & Sigara sp. & 22 & Ps-M-D & Se & G4 \\
\hline & Corixidae (ninfas) & 28 & Hm-M-D Pa & $\mathrm{Se}-\mathrm{Hu}$ & G4 \\
\hline & Thermonectus sp. & 6 & $\mathrm{M}$ & Se & G4 \\
\hline & Dysticidae (larvas) & 11 & $\mathrm{Hm}$ & Se & G4 \\
\hline & Derallus sp. & 6 & $\mathrm{M}$ & $\mathrm{Se}$ & G4 \\
\hline & Enochrus sp. & 22 & Pa-Ps-M-D & $\mathrm{Se}$ & G4 \\
\hline & Paracymus sp. & 11 & M-D & Se & G4 \\
\hline & Lepidoptera & 6 & D & $\mathrm{Hu}$ & G4 \\
\hline & Psychodidae & 11 & $\mathrm{~Pa}$ & $\mathrm{Se}-\mathrm{Hu}$ & $\mathrm{G} 2$ \\
\hline & Culicidae & 61 & Hm-M-Pa- D & $\mathrm{Se}-\mathrm{Hu}$ & G3 \\
\hline & Ceratopogonidae & 6 & $\mathrm{~S}$ & Se & $\mathrm{G} 2$ \\
\hline & Stratiomydae & 56 & Hm-Ps-M-D-Pa & $\mathrm{Se}-\mathrm{Hu}$ & $\mathrm{G} 2$ \\
\hline & Ephydridae & 22 & Hm-Ps-M & Se & G4 \\
\hline & Muscidae & 6 & $M$ & Se & G4 \\
\hline & Chironominae & 83 & Hm-Ps-M-S-Pa-D & $\mathrm{Se}-\mathrm{Hu}$ & G2 \\
\hline & Tanypodinae & 61 & Hm-Ps-M-S-D-Pa & $\mathrm{Se}-\mathrm{Hu}$ & G4 \\
\hline
\end{tabular}




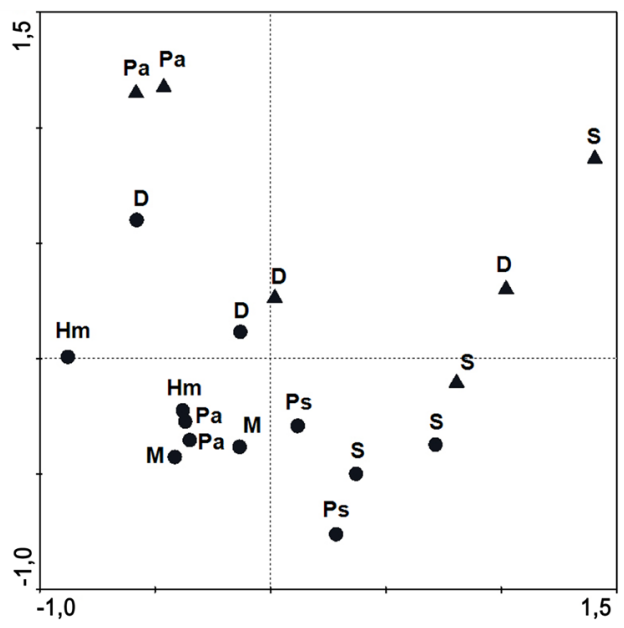

Figura 2. Análisis de Correspondencias (AC) de las muestras de macroinvertebrados colectadas en diferentes hábitats de la charca temporaria durante el período seco (círculos) y húmedo (triángulos). Pa: Polygonum aviculare, Hm: Heteranthera multiflora, Ps: Pistia stratiotes, M: macroalgas, S: sedimento sin vegetación, D: sector de descarga del desagüe.

Figure 2. Correspondence analysis (CA) of macroinvertebrates samples collected in different habitats of the temporary pond during the dry (circles) and wet period (triangles). Pa: Polygonum aviculare, Hm: Heteranthera multiflora, Ps: Pistia stratiotes, M: macroalgae, S: sediment without vegetation, D: drain discharge sector.
Tabla 3. Taxa indicadores (VI > 70\%) para cada hábitat de la charca temporaria (Río Cuarto, Córdoba) durante el período seco y húmedo. Vl: valor indicador

Table 3. Indicators taxa (IV > 70\%) for each habitat of the temporary pond (Río Cuarto, Córdoba) during the dry and wet period. IV: indicator value.

\begin{tabular}{|c|c|c|c|}
\hline PERÍODO SECO & & PERÍODO HÚMEDO & \\
\hline Hábitats & VI & Hábitats & VI \\
\hline Polygonum aviculare & & Polygonum aviculare & \\
\hline Liodessus sp. & 88,5 & Belostomidae (ninfas) & 100 \\
\hline Heteranthera multiflora & & Corixidae (ninfas) & 100 \\
\hline Dysticidae (larvas) & 100 & Entomobryidae & 100 \\
\hline Dactylobiotus partenogeneticus & 99,6 & Sminthuridae & 100 \\
\hline Aeshnidae & 94,6 & Isotomidae & 97,9 \\
\hline Ostracoda & 83,9 & Ostracoda & 97,4 \\
\hline Macroalgas & & Culicidae & 90,3 \\
\hline Stylaria sp. & 95,7 & Coenagrionidae & 89,4 \\
\hline Descarga del desagüe & & Belostoma sp. & 70,8 \\
\hline Poduridae & 94,5 & Sedimento sin vegetación & \\
\hline Sminthuridae & 78,3 & Naidinae & 97,3 \\
\hline \multirow[t]{3}{*}{ Culicidae } & 78,1 & Nematoda & 94,3 \\
\hline & & Descarga del desagüe & \\
\hline & & Chironominae & 88 \\
\hline
\end{tabular}

72 \% (Nematoda, Tanypodinae, Pristina sp., Stratiomyidae, Chironominae, de mayor a menor respectivamente). Por el contrario, Naidinae fue el único taxón indicador del período húmedo ( $\mathrm{VI}=86 \%)$.

El número de taxa del G3 de estrategia de vida

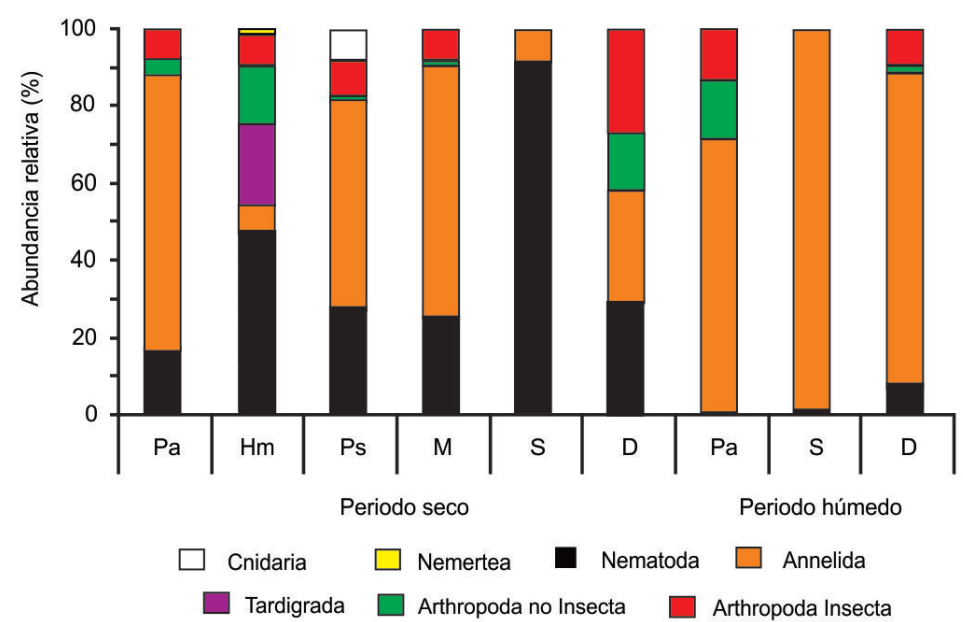

Figura 3. Abundancia relativa de los principales grupos taxonómicos en los diferentes hábitats de la charca temporaria durante el período seco y húmedo. Pa: Polygonum aviculare, Hm: Heteranthera multiflora, Ps: Pistia stratiotes, M: macroalgas, S: sedimento sin vegetación, D: sector de descarga del desagüe.

Figure 3. Relative abundance of the main taxonomic groups in the different habitats of the temporary pond during the dry and wet period. Pa: Polygonum aviculare, Hm: Heteranthera multiflora, Ps: Pistia stratiotes, M: macroalgae, S: sediment without vegetation, D: drain discharge sector. 
Tabla 4. Resultados de los ANOVAs de una vía aplicados para comparar el número de taxa dentro de cada grupo de estrategia de vida entre los distintos hábitats del período seco y húmedo. Los análisis se realizaron por período hidrológico. Los valores $p$ significativos $(p<0.05)$ se muestran en negrita. $F$ : estadístico F de Fisher, gl: grados de libertad, (factor, error).

Table 4. One-way ANOVA results applied to compare the taxa number in each life strategy group among the different habitats of the dry and wet period. Analyses were performed for each hydrological period. Significant values $(p<0.05)$ are in bold. F: Fisher statistic, gl: degree of freedom.

\begin{tabular}{cccccccc}
\hline & \multicolumn{3}{c}{ PERÍODO SECO } & \multicolumn{4}{c}{ PERíODO HÚMEDO } \\
\hline & gl & $\mathbf{F}$ & $\mathbf{p}$ & $\mathbf{g l}$ & $\mathbf{F}$ & $\mathbf{p}$ \\
\hline Grupo 1 & 5,6 & 1,98 & 0,215 & Grupo 1 & 2,3 & 3,50 & 0,164 \\
Grupo 2 & 5,6 & 1,07 & 0,461 & Grupo 2 & 2,3 & 0,60 & 0,604 \\
Grupo 3 & 5,6 & 4,80 & $\mathbf{0 , 0 4 1}$ & Grupo 3 & 2,3 & 3,00 & 0,192 \\
Grupo 4 & 5,6 & 2,15 & 0,189 & Grupo 4 & 2, 3 & 21,90 & 0,016 \\
\hline
\end{tabular}

fue diferente entre los hábitats del período seco, de acuerdo con los resultados del ANOVA (Tabla 4, Figura 4). Los hábitats $P$. stratiotes y sedimento sin vegetación no registraron taxa pertenecientes al G3 (prueba DGC a posteriori, $p<0,05)$. Durante el período húmedo, el número de taxa del G4 fue mayor en el hábitat $P$. aviculare, con respecto a los otros dos hábitats de ese período (prueba DGC a posteriori, $\mathrm{p}<0,05$ ) (Tabla 4, Figura 4). Durante este período, los hábitats en sector de descarga del desagüe y $P$. aviculare presentaron todos los grupos de estrategia de vida mientras que el sedimento sin vegetación sólo presentó taxa pertenecientes al G1 (Figura 4).

Los ANOVAs de dos vías mostraron diferencias en los atributos estructurales de la comunidad entre los distintos hábitats considerados. El sedimento sin vegetación fue el hábitat que presentó los menores valores de diversidad, equitatividad y riqueza y la mayor abundancia

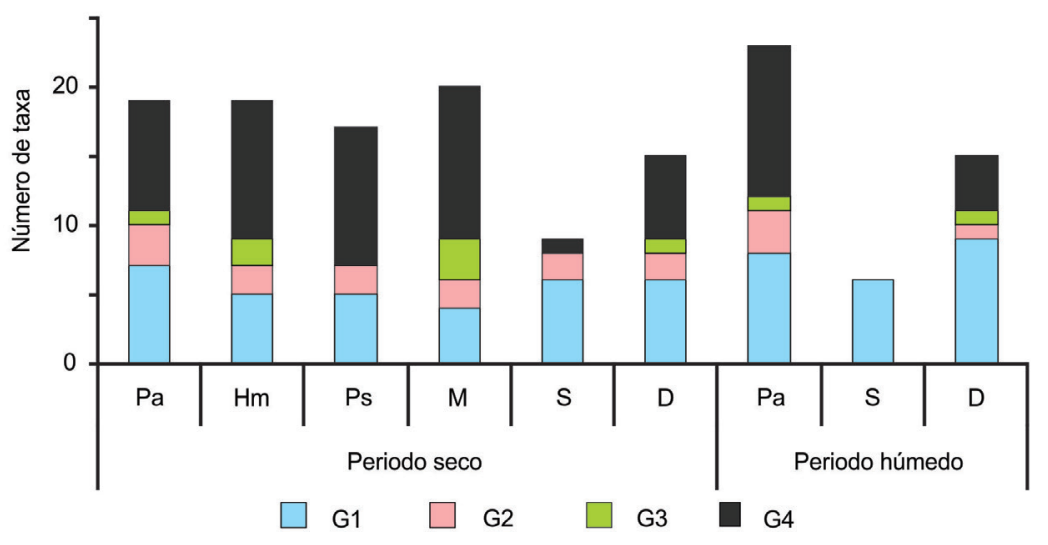

Figura 4. Número de taxa en cada grupo de estrategia de vida en los diferentes hábitats durante el período seco y húmedo. Pa: Polygonum aviculare, Hm: Heteranthera multiflora, Ps: Pistia stratiotes, M: macroalgas, S: sedimento sin vegetación, D: sector de descarga del desagüe. Grupos de estrategias de vida: G1: sobreviven a la desecación, tienen dispersión pasiva, necesitan agua para la reproducción; G2: sobreviven a la desecación, tienen dispersión activa, necesitan agua para la reproducción; G3: sobreviven a la desecación, tienen dispersión activa, no necesitan agua para la reproducción; G4: no sobreviven a la desecación, tienen dispersión activa, necesitan agua para la reproducción.

Figure 4. Taxa number of each life strategy group in the different habitats during the dry and wet period. Pa: Polygonum aviculare, Hm: Heteranthera multiflora, Ps: Pistia stratiotes, M: macroalgae, S: sediment without vegetation, D: drain discharge sector. Life strategy groups: G1: survive desiccation, have passive dispersion, need water for reproduction; G2: survive desiccation, have active dispersion, need water for reproduction; G3: survive desiccation, have active dispersion, do not need water for reproduction; G4: do not survive desiccation, have active dispersion, need water for reproduction. 
Tabla 5. Resultados de los ANOVAs de dos vías aplicados para comparar los atributos estructurales del ensamble de macroinvertebrados entre los distintos hábitats y períodos hidrológicos. Los valores $p$ significativos $(p<0.05)$ se muestran en negrita. F: estadístico F de Fisher, gl: grados de libertad.

Table 5. Two-way ANOVA results applied to compare the structural attributes of macroinvertebrate assemblages among the different habitats and hydrological periods. Significant values $(p<0.05)$ are in bold. F: Fisher statistic, gl: degree of freedom.

\begin{tabular}{|c|c|c|c|c|c|c|c|}
\hline & $\begin{array}{c}\mathbf{g l} \\
\text { (factor, error) }\end{array}$ & $F$ & $p$ & & $\begin{array}{c}\mathrm{gl} \\
\text { (factor, error) }\end{array}$ & $F$ & $p$ \\
\hline DIVERSIDAD & & & & EQUITATIVIDAD & & & \\
\hline Período & 1,6 & 3,77 & 0,100 & Período & 1,6 & 9,44 & 0,021 \\
\hline Hábitat & 2,6 & $\begin{array}{r}33,2 \\
1\end{array}$ & 0,001 & Hábitat & 2,6 & 50,61 & 0,001 \\
\hline Período*Hábitat & 2,6 & 1,23 & 0,357 & Período*Hábitat & 2,6 & 3,11 & 0,119 \\
\hline RIQUEZA & & & & ABUNDANCIA & & & \\
\hline Período & 1,6 & 9,42 & 0,022 & Período & 1,6 & 4,46 & 0,079 \\
\hline Hábitat & 2,6 & $\begin{array}{r}50,5 \\
0\end{array}$ & 0,001 & Hábitat & 2,6 & 40,38 & 0,001 \\
\hline Período*Hábitat & 2,6 & 3,10 & 0,119 & Período*Hábitat & 2,6 & 0,01 & 0,992 \\
\hline
\end{tabular}

de invertebrados (Tabla 5, Figura 5, prueba DGC a posteriori, $\mathrm{p}<0,05)$. La diversidad, la equitatividad y la riqueza fueron mayores en el sector de descarga del desagüe y en los parches de $P$. aviculare (Figura 5). La equitatividad y riqueza taxonómica también variaron entre
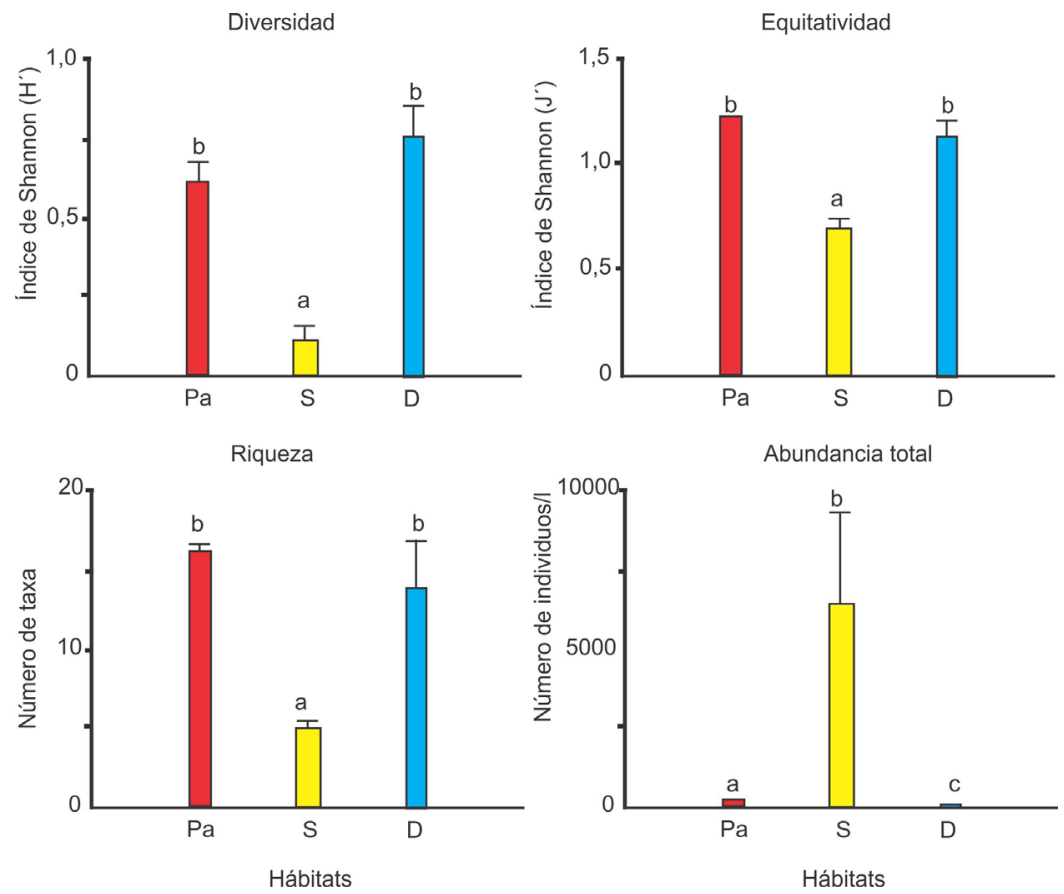

Figura 5. Variación de los atributos estructurales diversidad de Shannon, equitatividad, riqueza taxonómica y abundancia total de invertebrados entre los hábitats de la charca temporaria muestreados en ambos períodos hidrológicos. Las letras sobre las barras representan las diferencias de acuerdo a la prueba a posteriori DGC del ANOVA de dos vías $(\mathrm{p}<0,05)$. Pa: Polygonum aviculare, S: sedimento sin vegetación, D: sector de descarga del desagüe.

Figure 5. Variation of the structural attributes Shannon diversity (A), evenness (B), taxonomic richness $(C)$ and total abundance of invertebrates (D) among habitats of the temporary pond sampled in both hydrological periods. Letters on bars represent the differences according to the DGC post-hoc test of the two-way ANOVA $(p<0.05)$. Pa: Polygonum aviculare, S: sediment without vegetation, D: drain discharge sector. 
períodos hidrológicos correspondiendo los valores más elevados al período seco (Tabla 5 , prueba DGC a posteriori, $\mathrm{p}<0,05)$.

\section{DISCUSIÓN}

Los macroinvertebrados acuáticos registrados mostraron una segregación espacial y temporal en la charca temporaria. El Análisis de Correspondencias mostró una separación de las muestras en función del hábitat y del período hidrológico, indicando una diferenciación de los ensambles de macroinvertebrados en relación con la presencia de macrófitas acuáticas y con la estacionalidad. La segregación del ensamble vinculada con la presencia de vegetación acuática concuerda con los resultados obtenidos por otros autores (Bazzanti et al., 2010; Della Bella et al., 2005; Solimini et al., 2005). Las plantas acuáticas, en comparación a los ambientes no vegetados, incrementan la heterogeneidad del hábitat influyendo en la composición y estructura de la comunidad de macroinvertebrados (Bazzanti et al., 2009; 2010; Fontanarrosa et al., 2013). Por otro lado, la segregación de las muestras en relación con el período hidrológico demuestra una sucesión en la comunidad de macroinvertebrados durante el ciclo estacional, en coincidencia con lo reportado por otros estudios (Bazzanti et al., 1996; Boix et al., 2004; Culioli et al., 2006). Esta segregación temporal de la comunidad se relaciona con las diferentes estrategias de historia de vida de los organismos, tales como dispersión o resistencia a la sequía (Wiggins et al., 1980), y también con los cambios en la comunidad vinculados a la estructura trófica (Boix et al., 2004).

Durante el período seco, Liodessus sp. (Coleoptera) fue el taxón indicador del hábitat $P$. aviculare; mientras que en $\mathrm{H}$. multiflora los mayores valores indicadores se obtuvieron para larvas de Dytiscidae (Coleoptera), el tardígrado D. parthenogeneticus y Aeshnidae (Odonata). Los coleópteros Dytiscidae, dentro de los cuales se incluye Liodessus, utilizan las macrófitas como sitios de oviposición y refugio (Bazzanti et al., 2010; Fontanarrosa et al., 2013). Por otra parte, el tardígrado $D$. parthenogeneticus vive preferentemente en ambientes lénticos sobre algas filamentosas o macrófitas alimentándose de sus fluidos (Garey et al., 2008). Asimismo, las especies de Aeshnidae han sido reportadas en estrecha asociación a la vegetación acuática desde donde acechan a sus presas (Westfall \& Tennessen, 1996).

Durante el período húmedo, estados inmaduros de Corixidae y Belostomatidae (Heteroptera) y colémbolos Entomobryidae y Sminthuridae presentaron los mayores valores indicadores en $P$. aviculare. Los coríxidos y belostomátidos son considerados pioneros al inicio de la estación húmeda (Fischer et al., 2000) ya que son capaces de distinguir hábitats acuáticos de pequeño tamaño para la oviposición durante el vuelo (Boda \& Csabai, 2009). Asimismo, los heterópteros acuáticos son organismos depredadores estrechamente relacionados con las macrófitas que les proveen refugio y alimento (Jara et al., 2013). Por otro lado, Chironominae fue el único taxón indicador en el sector de la descarga del desagüe, y al igual que los coríxidos y belostomátidos, es considerado pionero en las charcas temporales (Epele \& Miserendino, 2016). Estos dípteros se caracterizan por poseer una alta fecundidad, ciclos de vida cortos y capacidad para resistir como larvas o huevos a la fase seca de las charcas (Bazzanti et al., 1997; Wiggins et al., 1980) lo que les permite formar abundantes poblaciones al retornar las condiciones favorables.

El análisis temporal de los grupos taxonómicos dominantes y el método IndVal demostraron que Nematoda fue característico del período seco, mientras que Naidinae fue dominante durante el período húmedo. La estacionalidad implica una sucesión de períodos de circulación y estancamiento del agua con diferentes temperaturas, contenido de oxígeno y minerales (Collado \& Schmelz, 2001). En consecuencia, se producen cambios en la composición del sustrato especialmente en términos de contenido y calidad de la materia orgánica. Los resultados de estos procesos suponen un recambio de los recursos alimentarios para oligoquetos y nemátodos (Majdi \& Traunspurger, 2015), lo que podría explicar la alternancia estacional en la dominancia de estos invertebrados.

El análisis de los grupos de estrategia de vida indicó que los Grupos 1 y 4 presentaron el mayor número de taxa en casi todos los hábitats en ambos períodos hidrológicos, lo 
que coincide con Bazzanti et al. (2009, 2010). Los taxa pertenecientes al Grupo 1 como anélidos y nemátodos se asociaron principalmente al sedimento sin vegetación; mientras que una gran abundancia de taxa del Grupo 4 como heterópteros, coleópteros y odonatos se registró en los hábitats vegetados. Los organismos residentes (o colonizadores pasivos) pertenecientes al Grupo 1, se encuentran comúnmente en los sedimentos no vegetados ya que resisten la desecación a través de huevos, larvas y adultos (Wiggins et al., 1980; Williams, 1997; Bazzanti et al., 2010). Por el contrario, la asociación observada entre los taxa del Grupo 4 y los hábitats vegetados puede interpretarse considerando que estos hábitats son más estables y aumentan el área superficial disponible para los taxa móviles de este grupo que no sobreviven a la desecación (Bazzanti et al., 2009).

Los atributos estructurales del ensamble presentaron diferencias entre los hábitats. La mayor diversidad se registró en el sector de descarga del desagüe y la mayor equitatividad y riqueza taxonómica en el hábitat vegetado $P$. aviculare. Cabe destacar que los márgenes de la charca en el sector de descarga del desagüe presentaban densos parches de $P$. aviculare. Estos resultados concuerdan con otros estudios que también señalan valores elevados de riqueza y diversidad en los ambientes vegetados vinculados a la heterogeneidad y los recursos de calidad que proveen estos hábitats (Heino, 2000; Della Bella et al., 2005; Bazzanti et al., 2010). Por el contrario, el fondo sin vegetación presentó la mayor abundancia de invertebrados, dada principalmente por nemátodos. Estos metazoos son muy abundantes en los sedimentos acuáticos, ya que pueden soportar condiciones desfavorables como por ejemplo bajas concentraciones de oxígeno (Abebe et al., 2008; Tahseen, 2012). Los nemátodos en las charcas temporales entran en quiescencia en respuesta al estrés hídrico (Wiggins et al., 1980) y junto a la capacidad de autofecundación y la dispersión pasiva, se benefician frente a estas condiciones desfavorables (Tahseen, 2012).

La equitatividad y la riqueza taxonómica también difirieron entre los períodos hidrológicos, registrándose los valores más elevados en el período seco (junio), luego de seis meses de permanencia del agua. En contraste, los valores correspondientes al período húmedo se obtuvieron luego de tres meses del inicio de la época de lluvias. Si bien, en este estudio se recolectaron muestras en una fecha de cada período, los resultados sugieren una influencia de la longitud del hidroperíodo en la riqueza taxonómica, de manera similar a lo reportado por otros estudios (Bazzanti et al., 2009; 2010; Della Bella et al., 2005). En hidroperíodos más largos, un mayor número de especies es capaz de completar su desarrollo y mantener poblaciones viables, especialmente aquellas que presentan dispersión activa ya que la permanencia del agua proporciona mayor tiempo disponible para la colonización (Fontanarrosa et al., 2009). Futuros estudios que incluyan mayor frecuencia de muestreos en cada período en diferentes charcas de la región permitirán profundizar la información acerca del efecto de las fluctuaciones estacionales en la permanencia del agua sobre el ensamble de macroinvertebrados. Los resultados obtenidos ratifican la importancia de las macrófitas como elemento clave en el mantenimiento de la biodiversidad, proporcionando información de base con uso potencial en el monitoreo, el manejo y la conservación de la charca temporaria urbana "Las Brujas".

\section{AGRADECIMIENTOS}

Agradecemos a Javier Márquez y Cintia Barroso por su ayuda en las tareas de campo y laboratorio y a la Dra. Mariana Rocha por la identificación de los ejemplares de Tardigrada. Agradecemos también los comentarios de los revisores los cuales mejoraron la primera versión del manuscrito. Este trabajo fue financiado por la Secretaría de Ciencia y Técnica de la UNRC. Deseamos agradecer especialmente a la profesora Gabriela Villalba por su ayuda en las tareas de campo y laboratorio y dedicamos este trabajo a su memoria.

\section{REFERENCIAS}

Abebe, E., Decraemer, W. \& De Ley, P. (2008). Global diversity of nematodes (Nematoda) in freshwater. Hydrobiologia, 595, 67-78. 
APHA. (1998). Standard methods for examination of water and wastewater. 20th ed. American Public Health Association, American Water Works Association and the Water and Environment Federation. Washington (DC).

Barbour, M. T., Stribling, J. B. \& Karr, J. R. (1995). Multimetric approach for establishing biocriteria and measuring biological conditions. In: Davis, W. S. \& Simon, T. P. (Eds.). Biological Assessment and Criteria: Tools for Water Resource Planning and Decision Making (pp. 63-77). Boca Raton, FL: Lewis Publishers.

Bazzanti, M., Baldoni, S. \& Seminara, M. (1996). Invertebrate macrofauna of a temporary ponds in Central Italy: composition, community parameters and temporal succession. Archiv für Hydrobiologie, 137,77-94.

Bazzanti, M., Seminara, M. \& Baldoni, S. (1997). Chironomids (Diptera: Chironomidae) from three temporary ponds of different wet phase duration in central Italy. Journal of Freshwater Ecology, 12(1), 89-99.

Bazzanti, M., Della Bella, V. \& Grezzi, F. (2009). Functional characteristics of macroinvertebrate communities in Mediterranean ponds (Central Italy): influence of water permanence and mesohabitat type. Annales de Limnologie International Journal of Limnology, 45(1): 29-39.

Bazzanti, M., Coccia, C. \& Dowgiallo, M. G. (2010). Microdistribution of macroinvertebrates in a temporary pond of Central Italy: taxonomic and functional analyses. Limnologica, 40(4), 291-299.

Boda, P. \& Csabai, Z. (2009). Seasonal and diel dispersal activity characteristics of Sigara lateralis (Leach, 1817) (Heteroptera: Corixidae) with special emphasis on possible environmental factors and breeding state. Aquatic Insects, 31(4), 301-314.

Boix, D., Sala, J., Quintana, X. D. \& Moreno-Amich R. (2004). Succession of animal community in a Mediterranean temporary pond. Journal North American Benthological Society, 23(1), 29-49.

Boix, D., Gascón, S., Sala, J., Martinoy, M., Gifre, J. \& Quintana, X. D. (2005). A new index of water quality assessment in Mediterranean wetlands based on crustacean and insect assemblages: the case of Catalunya (NE Iberian Peninsula). Aquatic Conservation: Marine and Freshwater Ecosystems, 15(6), 635-651.

Boix, D., Kneitel, J., Robson, B. J., Duchet, C., Zúñiga, L., Day, J., Gascón, S., Sala, J., Quintana, X.D. \& Blaustein, L. (2016). Invertebrates of freshwater temporary ponds in Mediterranean climates. In: Batzer, D. \& Boix D. (eds.), Invertebrates in freshwater wetlands (pp. 141-189). Switzerland: Springer.

Cabido, D., Cabido, M., Garré, S. M., Gorgas, J. A., Miatello, R., Ravelo, A., Rambaldi, S. y Tassile, J. L. (2003). Regiones Naturales de la provincia de Córdoba. Agencia Córdoba DACyT. Dirección de Ambiente. Gobierno de la Provincia de Córdoba. Argentina.

Céréghino, R., Biggs, J., Declerck, S. \& Oertli, B. (2008). The ecology of European ponds: defining the characteristics of a neglected freshwater habitat. Hydrobiologia, 597,1-6.

Collado, R. \& Schmelz, M. (2001). Oligochaete distribution patterns in two German hardwater lakes of different trophic state. Limnologica, 31(4), 317-328.

Culioli, J. L., Foata, J., Mori, C., Orsini, A. \& Marchand, B. (2006). Temporal succession of the macroinvertebrate fauna in a corsican temporary pond. Viet et Milieu-Life and Environment, 56(3), 215-221.

Della Bella, V., Bazzanti, M. \& Chiarotti, F. (2005). Macroinvertebrate diversity and conservation status of Mediterranean ponds in Italy: water permanence and mesohabitat influence. Aquatic Conservation: Marine and Freshwater Ecosystems, 15(6), 583-600.

Di Rienzo, J. A., Guzmán, A. W. \& Casanoves, F. (2002). A multiple comparisons method based on the distribution of the root node distance of a binary tree. Journal of Agricultural, Biological, and Environmental Statistics, 7(2), 129-142.

Di Rienzo, J. A., Casanoves, F., Balzarini, M. G., Gonzalez, L., Tablada, M. y Robledo, C. W. (2010). InfoStat versión 2010. Grupo InfoStat, F.C.A, Universidad Nacional de Córdoba, Argentina. Web link: http://www.infostat.com. ar. 
Domínguez, E. y Fernández, H. R. (Eds.). (2009). Macroinvertebrados bentónicos sudamericanos: Sistemática y biología. Fundación Miguel Lillo, Tucumán, Argentina.

Dufrêne, M. \& Legendre, P. (1997). Species assemblages and indicator species: The need for a flexible asymmetrical approach. Ecological Monographs, 67(3), 345-366.

Epele, L. B. \& Miserendino, M. L. (2016). Temporal dynamics of invertebrate and aquatic plant communities at three intermittent ponds in livestock grazed Patagonian wetlands. Journal Natural History, 50(11-12), 711-730.

European Pond Conservation Network, EPCN. (2008). Manifiesto sobre las charcas. Disponible en: https://www.europeanponds. org/publications/epcn-publications/ (fecha de acceso: 20-05-2020).

Fischer, S., Marinone, M. C., Fontanarrosa, M. S., Nieves, M. \& Schweigmann, N. (2000). Urban rain pools: seasonal dynamics and entomofauna in a park of Buenos Aires. Hydrobiologia, 441(1), 45-53.

Fontanarrosa, M. S., Collantes, M. B. \& Bachmann, A. O. (2009). Seasonal patterns of the insect community structure in urban rain pools of temperate Argentina. Journal of Insect Science, 9(10): 1-17.

Fontanarrosa, M. S., Collantes, M. B. \& Bachmann, A. O. (2013). Aquatic Insect Assemblages of Man-Made Permanent Ponds, Buenos Aires City, Argentina. Neotropical Entomology, 42(1): 22-31.

Garey, J. R., McInnes, S. J. \& Nichols, P. B. (2008). Global diversity of tardigrades (Tardigrada) in freshwater. Hydrobiologia, 595, 101-106.

Hassall C. (2014). The ecology of urban ponds. WIREs Water, 1,187-206.

Heino, J. (2000). Lentic macroinvertebrate assemblage structure along gradients in spatial heterogeneity, habitat size and water chemistry. Hydrobiologia, 418, 229-242.

Jara, F. G., Úbeda, C. A. \& Perotti, M. G. (2013). Predatory insects in lentic freshwater habitats from northwest Patagonia: richness and phenology. Journal Natural History, 47, 27492768.

Jeffries, M. J. (2003). Idiosyncratic relationships between pond invertebrates and environmental, temporal and patch specific predictors of incidence. Ecography, 26, 311-324.

Johansson, F. \& Suhling, F. (2004). Behaviour and growth of dragonfly larvae along a permanent to temporary water habitat gradient. Ecological Entomology, 29, 196-203.

Lee, A. C. K. \& Maheswaran, R. (2011). The health benefits of urban green spaces: a review of the evidence. Journal of Public Health, 33(2), 212-222.

Lopretto, E. C. y Tell, G. (1995). Ecosistemas de aguas continentales. Metodologías para su estudio. Tomo I, II y III. La Plata, Argentina: Ediciones Sur.

Majdi, N. \& Traunspurger, W. (2015). Free-living nematodes in the freshwater food web: a review. Journal of Nematology, 47(1), 28-44.

McCune, B. \& Mefford, M. J. (1999). Multivariate Analysis of Ecological Data. Version 4.25. Oregon: MjM Software, Gleneden Beach.

Mitsch, W. J. \& Gosselink, J. G. (2000). The value of wetlands: importance of scale and landscape setting. Ecological Economics, 35, 25-33.

Morrone, J. J. (2014). Biogeographical regionalisation of the Neotropical region. Zootaxa, 3782, 1-110.

Nieto, C., Ovando, X. M. C., Loyola, R., Izquierdo, A., Romero, F., Molineri, C., Rodríguez, J., Rueda Martín, P., Fernández, H., Manzo, V. \& Miranda, M J. (2017). The role of macroinvertebrates for conservation of freshwater systems. Ecology and Evolution, 7(14), 5502-5513.

Noble A. \& Hassall C. (2015). Poor ecological quality of urban ponds in northern England: causes and consequences. Urban Ecosystems, 18(2), 649-662.

Ramsar Convention Secretariat. (2002). Guidance for identifying, sustainably managing, and designating temporary pools as Wetlands of International Importance. 8TH Meeting of the 
Conference of the Contracting Parties to the Convention on Wetlands. Resolution VIII.33. https://www.ramsar.org.

Solimini, A. G., Della Bella, V. \& Bazzanti, M. (2005). Macroinvertebrate size spectra of Mediterranean ponds with differing hydroperiod length. Aquatic Conservation: Marine and Freshwater Ecosystems, 15, 601-611.

Tahseen, Q. (2012). Nematodes in aquatic environments: adaptations and survival strategies. Biodiversity Journal, 3(1), 13-40.

ter Braak, C. J. F. \& Smilauer, P. (1998). CANOCO Reference manual and User's guide to Canoco for Windows: software for Canonical Community Ordination (version 4). New York: Microcomputer power.

Wentworth, C. K., (1922). A scale of grade and class terms for clastic sediments. The Journal of Geology, 30(5), 377-392.
Westfall Jr., M. J. \& Tennessen, K. J. (1996). Odonata. In: Merritt R. W. \&, Cummins K.W. (eds). An introduction to the aquatic insects of North America. 3rd edition (pp. 164-211). lowa: Kendall/Hunt Publishing Company.

Wiggins G.B., Mackay R.J. \& Smith I.M. (1980). Evolutionary and ecological strategies of animals in annual temporary pools. Archiv für Hydrobiologie, Supplement 58, 97-206.

Williams D. D. (1997). Temporary ponds and their invertebrate communities. Aquatic Conservation: Marine and Freshwater Ecosystems, 7, 105-117.

Williams P., Whitfield M., Biggs J., Bray S., Fox G., Nicolet P. \& Sear D. (2004). Comparative biodiversity of rivers, streams, ditches and ponds in an agricultural landscape in Southern England. Biological Conservation, 115, 329-341. 